\title{
High p16 Expression Is Associated with Malignancy and Shorter Disease-Free Survival Time in Solitary Fibrous Tumor/Hemangiopericytoma
}

\author{
Yuanxin Liang ${ }^{1}$ Robert S. Heller ${ }^{2} \quad$ Julian K. Wu ${ }^{2} \quad$ Carl B. Heilman ${ }^{2} \quad$ Arthur S. Tischler ${ }^{1} \quad$ Knarik Arkun $^{1}$ \\ ${ }^{1}$ Department of Pathology and Laboratory Medicine, Tufts Medical \\ Center and Tufts University School of Medicine, Boston, \\ Massachusetts, United States \\ ${ }^{2}$ Department of Neurosurgery, Tufts Medical Center and Tufts University \\ School of Medicine, Boston, Massachusetts, United States

\begin{abstract}
Address for correspondence Knarik Arkun, MD, Department of Pathology and Laboratory Medicine, Tufts Medical Center, 800 Washington Street, Boston, MA 02111, United States
\end{abstract} \\ (e-mail: karkun@tuftsmedicalcenter.org).

\begin{abstract}
Objective Solitary fibrous tumors (SFT) and hemangiopericytomas (HPC) are now classified along a single spectrum of fibroblastic mesenchymal tumors with NAB2-STAT6 fusion. This fusion acts as a driver mutation that constitutively activates EGR1, which is known to be involved in the $\mathrm{p} 16$ pathway. Overexpression of $\mathrm{p} 16$ is associated with malignancy and worse prognosis in multiple mesenchymal tumors. The authors sought to investigate p16 immunoexpression in association with malignancy and prognosis of SFT/HPC tumors.

Design Twenty-three SFT/HPC tumors (central nervous system [CNS]: 12, non CNS: 11) diagnosed at our institution from 2002 to 2016 were assigned into 3 grades. Data from microarray immunohistochemistry for STAT6, synaptophysin, CD56, chromogranin, SST2A, EGR1, Ki67, and p16, grade and survival were analyzed.

Results CNS SFT/HPCs tend to be malignant (grade 3; 67 vs. $18 \%, p=0.036$ ) and more likely to express synaptophysin ( 33 vs. $0 \%, p=0.035$ ) than non CNS tumors. Overexpression of p16 (immunopositivity $\geq 50 \%$ tumor cells) was associated with malignant (grade 3 ) tumors, and has a sensitivity of $70 \%(7 / 10)$, and a specificity of $77 \%(10 / 13)$, as a predictive marker for malignancy. SFT/HPC patients with low 16 expression demonstrated

Keywords

- solitary fibrous tumor

- hemangiopericytoma

- p16

- malignancy

- disease-free survival time

significantly longer disease-free survival time (median survival $>113$ months) than those with high p16 expression (median survival $=30$ months, $p=0.045$ ).

Conclusions SFT/HPCs in the CNS are more likely to be malignant than the tumors in other sites. High p16 expression is also associated with malignancy and shorter diseasefree survival time in SFT/HPC tumors in our study cohort. Clinically, p16 overexpression can be used as predictive marker for malignancy and prognosis and a possible therapeutic target.
\end{abstract}

\section{Introduction}

Solitary fibrous tumors (SFT) and hemangiopericytomas (HPC) are now considered along a single spectrum of neoplastic disease characterized by fibroblastic mesenchymal tumors with a rich, branching vascular pattern that can occur in any

received

May 6, 2018

accepted after revision

July 17,2018

published online

August 21, 2018

anatomic location. ${ }^{1}$ Both tumors share the same genomic inversion at chromosome $12 \mathrm{q} 13$ resulting in fusion of the NAB2 and STAT6 genes. ${ }^{2,3}$ The gene fusion inherits an activation domain from the signaling molecule STAT6, which converts a transcriptional repressor (NAB2) into a potent

(c) 2019 Georg Thieme Verlag KG Stuttgart · New York
DOI https://doi.org/ 10.1055/s-0038-1669419. ISSN 2193-6331. 
transcriptional activator (NAB2-STAT6) of EGR1. This fusion protein induces constitutive activation of EGR1, which regulates downstream gene expression as a transcription factor. ${ }^{4,5}$

Expression of the cell cycle checkpoint protein p16 (Ink4a, CDKN2a) is linked to induction of the transcription factor EGR1 in epidermal keratinocytes during malignant transformation in skin cells. Infection with retrovirus containing EGR1 significantly increases $\mathrm{p} 16$ promoter activity and elevates $\mathrm{p} 16$ protein levels in keratinocytes. ${ }^{6}$ Moreover, studies have shown that p16 overexpression is associated with malignancy and a worse prognosis in multiple mesenchymal tumors such as Ewing's sarcoma, ${ }^{7}$ adipocytic tumors, ${ }^{8}$ and myometrial tumors. ${ }^{9}$

To date, p16 expression and its clinical significance have not been studied in SFT/HPCs. In this study, we investigated p16 expression in 23 cases of SFT/HPC, focusing on the relationship of $\mathrm{p} 16$ expression to malignancy and prognosis.

\section{Materials and Methods}

The current study was approved by the Tufts Medical Center institutional review board. Twenty-three SFT/HPC tumors from 20 patients (2002-2016) with available formalin-fixed, paraffin-embedded (FFPE) blocks were included in this study. The diagnoses were confirmed by nuclear immunepositivity for STAT6 ( $n=22$ ) and by next generation sequencing identified NAB2-STAT6 fusion $(n=1)$. All cases were reviewed by 2 pathologists (K.A. and Y.L.) for diagnosis and grading. Clinical and radiographic records were reviewed for patient age, sex, tumor location, and recurrence data.

Two-tissue microarray paraffin blocks were prepared for immunohistochemistry (IHC). IHC was performed using anti-p16, synaptophysin, CD56, chromogranin, SST2A, and Ki67 antibodies (Ventana, Roche), and anti-EGR1 antibody (Abcam), following the standard protocol. STAT6 staining was performed as a send-out test at Massachusetts General Hospital. Assessment of IHC staining in tumors and controls was performed by 2 pathologists (K.A. and Y.L.). Positive staining for p16, EGR1, and Ki67 expression was evaluated in a quantitative fashion and expressed as a percentage of cell staining positive in the total population of tumor cells. The IHC results of the other markers were dichotomized as either positive or negative.

The tumors were assigned into 3 grades. Grade 1 tumors were those previously termed as benign SFT and grade 2 tumors were those previously termed as benign HPC. Grade 3 tumors, previously termed anaplastic HPC, were diagnosed on the basis of 5 or more mitoses per 10 high-powered field (HPF), hypercellularity, and necrosis. ${ }^{10,11}$ For the purposes of this study, cases were divided into a malignant group (grade 3 lesions) and a nonmalignant group (grade 1 and 2 lesions).

Values are presented as means and standard deviations (SD). The data was analyzed and graphed using Graphpad Prism 6. The unpaired, nonparametric $t$-test with 2 tails was used to compare the mean value differences between 2 groups; The $Z$-score test was used to compare the proportion differences between 2 groups. Fisher's test was used to evaluate the association between the rows and the columns in $2 \times 2$ contingency tables. ANOVA (analysis of variance) and multiple comparisons were used to compare differences among multiple groups. The Kaplan-Meier estimator was used for survival analysis; the log-rank (Mantel-Cox) test was used to compare with the Kaplan-Meier curves of different groups. $p<0.05$ was set as achieving statistical significance.

\section{Results}

At the time of tumor removal, the average patient-age was 56 years (range: $31-87$, median: 57 ). Fourteen tumors (61\%) were from male patients and 9 tumors (39\%) from female patients. In $12 / 23$ cases (52\%), tumors were located in the central nervous system (CNS) while the other 11 cases were outside of the CNS. Ten of 23 cases were classified as malignant (grade 3). The average tumor size was $5.4 \mathrm{~cm}$. Clinical information and IHC results are summarized in -Table 1.

\section{High p16 Expression Is Associated with Malignant SFT| HPCs}

Malignant (grade 3) SFT/HPCs harbored more p16 positive tumor cells than nonmalignant tumors (grades 1 and 2; average: $62 \%$ vs. $29 \%, p=0.034)$. Representative cases from grades 1, 2, and 3 are demonstrated in -Fig. 1. Malignant SFT/HPCs did not show a statistically significant higher Ki67 proliferative index than nonmalignant tumors (16\% vs. $9 \%, p=0.054$; - Table 1). Tumor size was not statistically associated with $\mathrm{p} 16$ expression $(R=-0.30, p=0.16)$. All SFT/HPCs in this study, showed at least a small subset of tumor cells population with p16 immunopositivity. High expression of p16 (determined using a cut-off of $50 \%$ of tumor cells positively staining on IHC) was significantly associated with malignancy ( $p=0.040$; - Fig. 1). As a predictive marker for malignancy, $\mathrm{p} 16$ expression has sensitivity of $70 \%(7 / 10)$ and specificity of $77 \%(10 / 13)$ in the study population.

\section{High p16 Expression Is Associated with Shorter Disease-Free Survival}

Patients with follow-up time $<12$ months or subtotal/partial resection were excluded for the disease-free survival analysis. At the time of the last follow-up, all of the patients included for the disease-free survival study $(n=15)$ were alive. Tumors recurred in 6 out of 15 cases (40\%). Nonmalignant SFT/HPC patients showed significantly longer disease-free survival time (median survival = 103 months) than malignant cases (median survival $=30$ months; $p=0.043$ ). SFT/HPC patients with low 16 expression demonstrated significantly longer diseasefree survival time (median survival $>113$ months) than those with high p16 expression (median survival = 30 months; $p=0.045$ ). The disease-free survival analysis also identified that patients with low p16 expression in nonmalignant SFT/ HPC had median survival $>113$ months; the patients with either malignant SFT/HPC or high $\mathrm{p} 16$ expression have median survival of 103 months, while the patients with high p16 expression and malignant SFT/HPC had median survival of only 30 months. (-Figs. 2A-C) 


\begin{tabular}{|c|c|c|c|c|c|c|c|c|c|c|c|c|c|c|c|c|c|c|c|c|c|c|c|}
\hline$\underset{\sim}{\mathbb{\Xi}}$ & ᄃ & ᄃ & 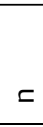 & c & ᄃ & $ᄃ$ & ᄃ & ᄃ & c & ᄃ & ᄃ & ᄃ & $\subset$ & 0 & $\subset$ & $ᄃ$ & ᄃ & 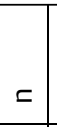 & 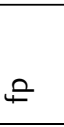 & 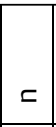 & ᄃ & $\subset$ & ᄃ \\
\hline 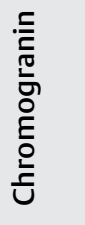 & $=$ & c & $=$ & 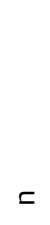 & c & c & $=$ & $=$ & c & $=$ & $=$ & c & $=$ & $=$ & 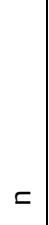 & $=$ & c & c & c & $=$ & $=$ & $=$ & c \\
\hline 号 & $\stackrel{2}{2}$ & 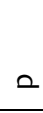 & 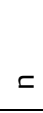 & 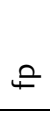 & ᄃ & $\frac{2}{3}$ & $\stackrel{2}{2}$ & $=$ & ᄃ & ᄃ & ᄃ & ᄃ & $c$ & 0 & $\circ$ & 0 & $\frac{2}{3}$ & $=$ & $\frac{n}{3}$ & 0 & ᄃ & $\subset$ & ᄃ \\
\hline 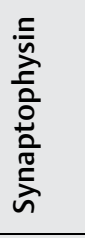 & ᄃ & ᄃ & 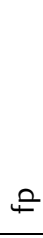 & ᄃ & $\subset$ & ᄃ & $\subset$ & ᄃ & ᄃ & $=$ & $=$ & ᄃ & $\subset$ & $=$ & 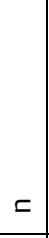 & ᄃ & 口 & 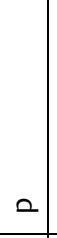 & $\cong$ & $=$ & ᄃ & $=$ & ᄃ \\
\hline$\frac{0}{\frac{1}{6}}$ & 0 & 0 & 0 & 0 & 0 & 0 & 0 & 0 & 口 & 0 & 0 & 0 & 3 & 0 & 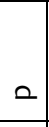 & 0 & 0 & 0 & 口 & 0 & 0 & 0 & ${ }^{\circ} \mathrm{C}$ \\
\hline 젛 & - & - & - & - & 0 & $m$ & $\nabla$ & $\sim$ & $N$ & $m$ & - & 0 & - & $m$ & 요 & - & เn & $N$ & 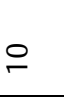 & 0 & $N$ & $\sim$ & 0 \\
\hline$\stackrel{\widehat{b}}{\underline{\underline{0}}}$ & 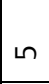 & & $\stackrel{\circ}{\circ}$ & 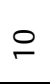 & & 움 & $\infty$ & Ln & & 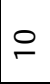 & $\Omega$ & $N$ & $\stackrel{\curvearrowright}{\sim}$ & $\stackrel{\circ}{-}$ & $\stackrel{\circ}{\longrightarrow}$ & 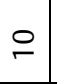 & ๒ & $\circ$ & $\stackrel{m}{m}$ & $\circ$ & $\stackrel{n}{\leftarrow}$ & $\simeq$ & \\
\hline
\end{tabular}

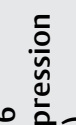

는 흘

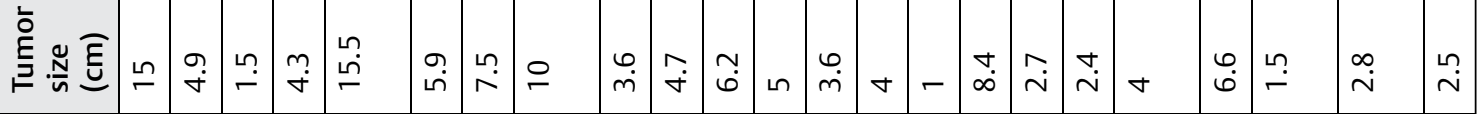

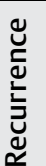

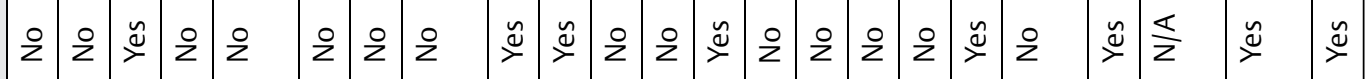

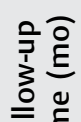

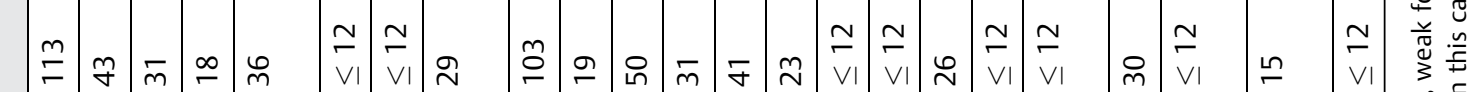

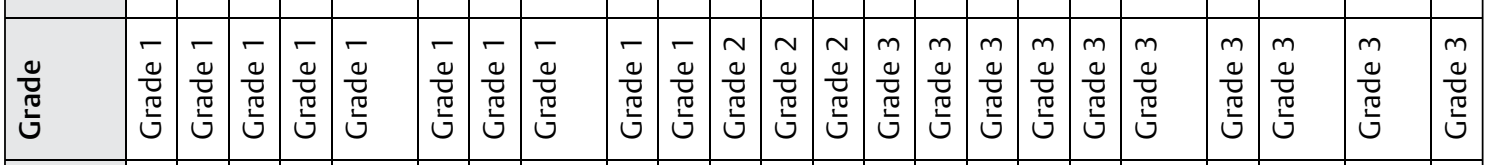

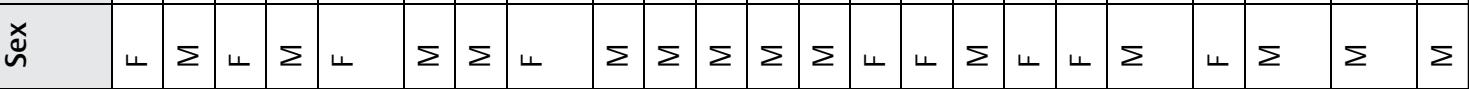

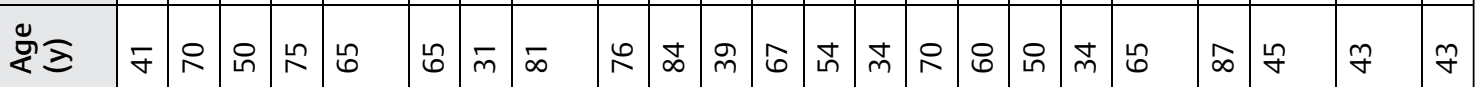

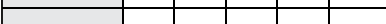



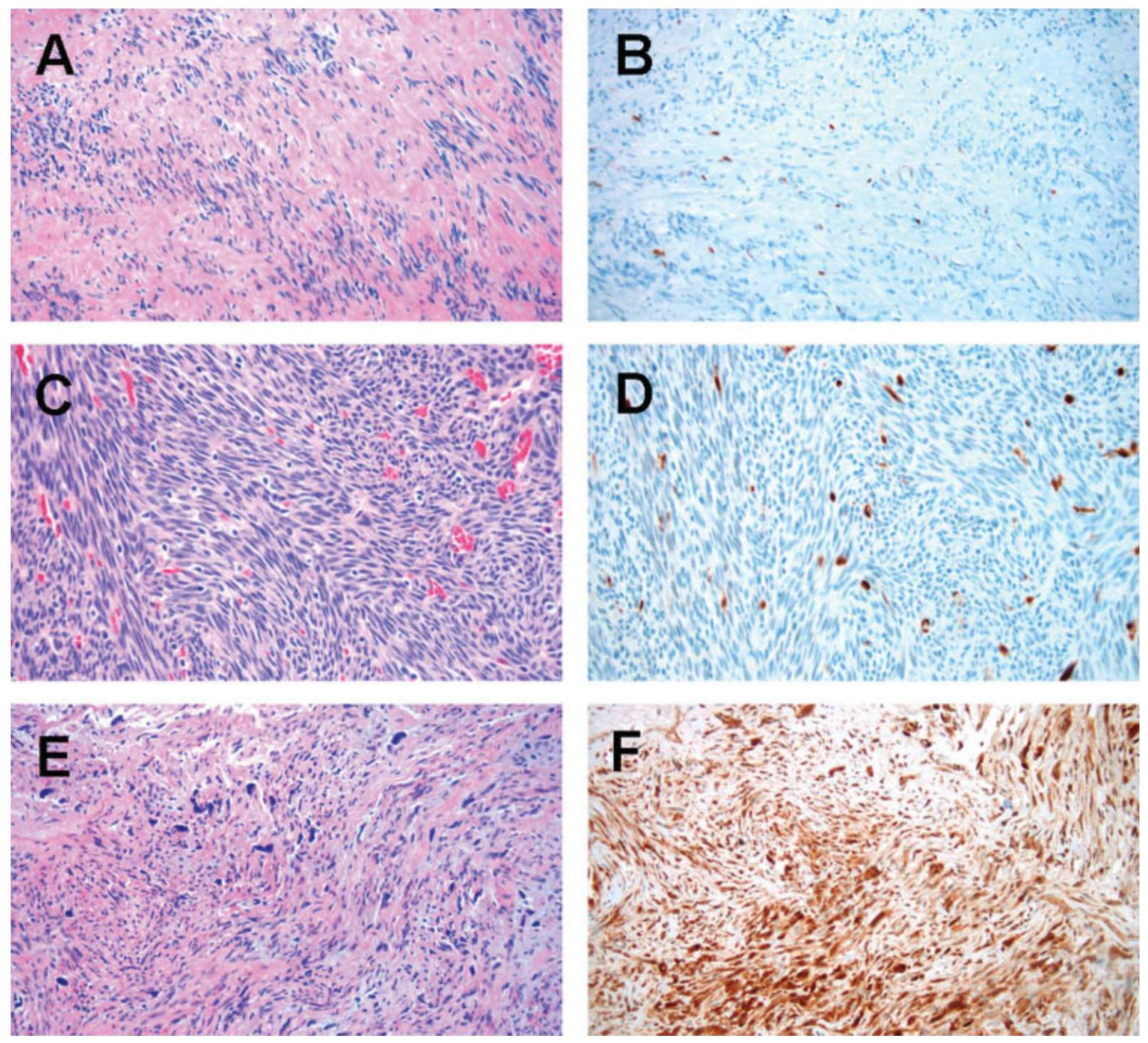

Fig. 1 Hematoxylin and eosin (H\&E) (left panels) and p16 expression (right panels) in SFT/HPCs tumors. (A, B): grade 1 tumor with low p16 expression, case \#2; (C, D): grade 2 tumor with low p16 expression, case \#11; (E, F): grade 3 tumor with high p16 expression, case \#20. 100× magnification.

\section{SFT/HPCs from the CNS Are More Likely to Be Malignant and to Express Synaptophysin}

More CNS SFT/HPCs were malignant (67\% vs. $18 \%, p=0.036$ ) and expressed synaptophysin ( $33 \%$ vs. $0 \%, p=0.035$ ) than non CNS SFT/HPCs. Chromogranin was negative in all tumors, but CD56 stained in 11 cases. None of these neuroendocrine markers show statistically significant correlation with malignancy, disease-free survival, or p16 expression. Two CNS tumors showed patchy focal staining with SST2A, while all other tumors were immunonegative. The tumor size of non CNS tumors was significantly larger than CNS SFT/HPCs (7.2 vs. $3.7 \mathrm{~cm}$ in average, $p=0.023$ ). In addition, the nonmalignant SFT/HPCs were significantly larger than malignant SFT/HPCs (6.7 vs. $3.6 \mathrm{~cm}$ in average, $p=0.048$ ). Patients with CNS SFT/ HPC demonstrated shorter disease-free survival time (median survival $=41$ months) than those with non CNS tumors (median survival = 103 months). However, the difference was not statistically significant ( $p=0.513$; - Fig. 2D).

\section{EGR1 Protein Is Rarely Detected in p16 Positive Tumor Cells by Immunohistochemistry}

Since all the cases in this study showed a population of $\mathrm{p} 16$ positive tumor cells, we sought to determine whether p16 expression resulted from NAB2-STAT6 fusion-activated EGR1. We evaluated p16, EGR1, and dual p16/EGR1 immunostains. There was no statistical correlation between the percentages of p16 positive cells and EGR1 positive cells (correlation value = $-0.1311, p=0.551)$. Interestingly, only rare cells expressed simultaneous p16 and EGR1 by dual immunostaining (- Fig. 3).

\section{Discussion}

Over the past decade, studies were trying to link hemangiopericytomas and solitary fibrous tumors. Most recently, STAT6-NAB2 fusion allowed to restructure SFT and HPC into one entity "SFT/HPC" and use grading system to differentiate between them. ${ }^{11}$ 

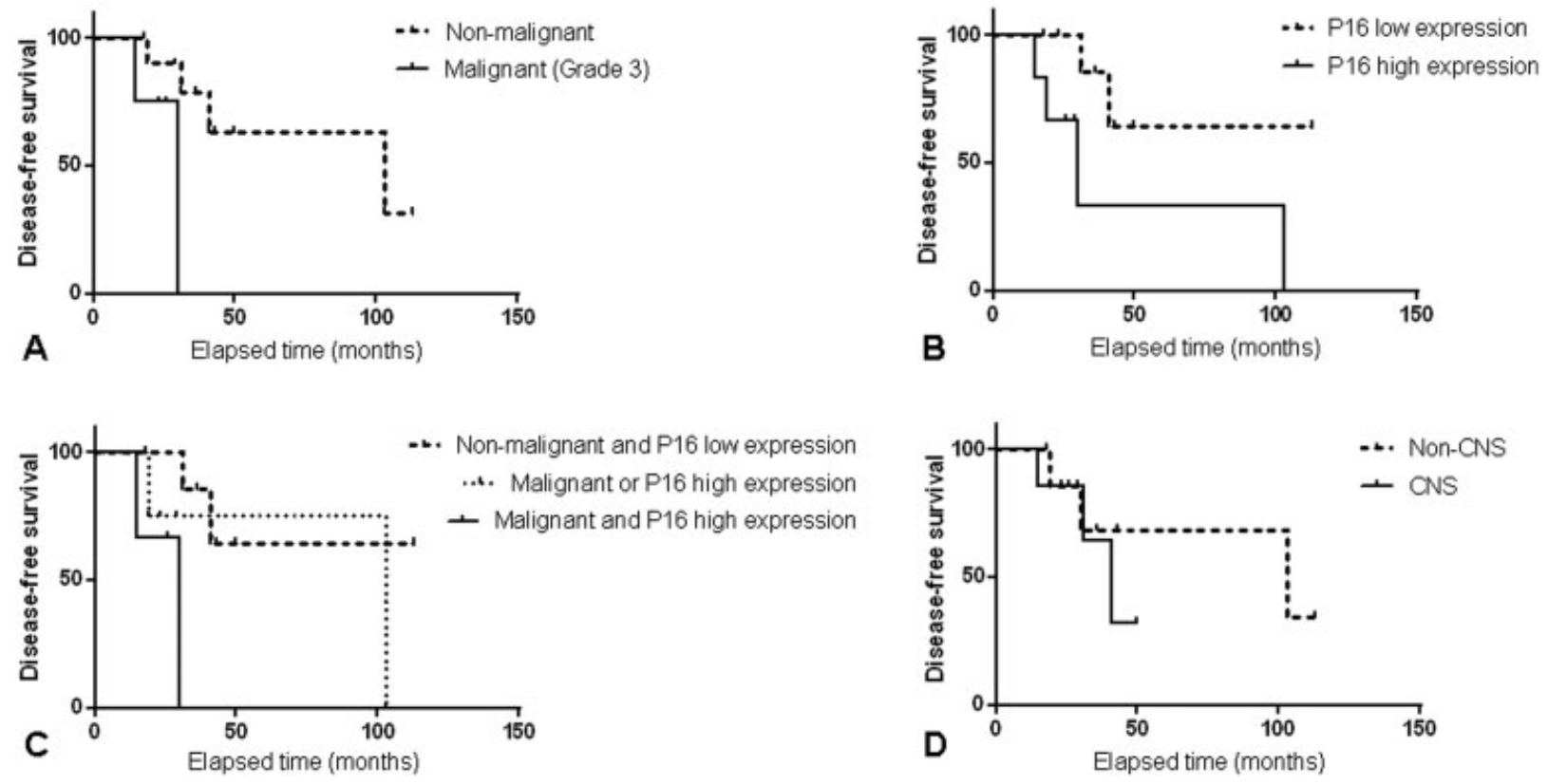

Fig. 2 Disease-free survival analysis [(A): Nonmalignant vs. malignant; (B): p16 low expression vs. p16 high expression; (C): Nonmalignant and p16 low expression vs. malignant or p16 high expression vs. malignant and p16 high expression; (D): non CNS vs. CNS].
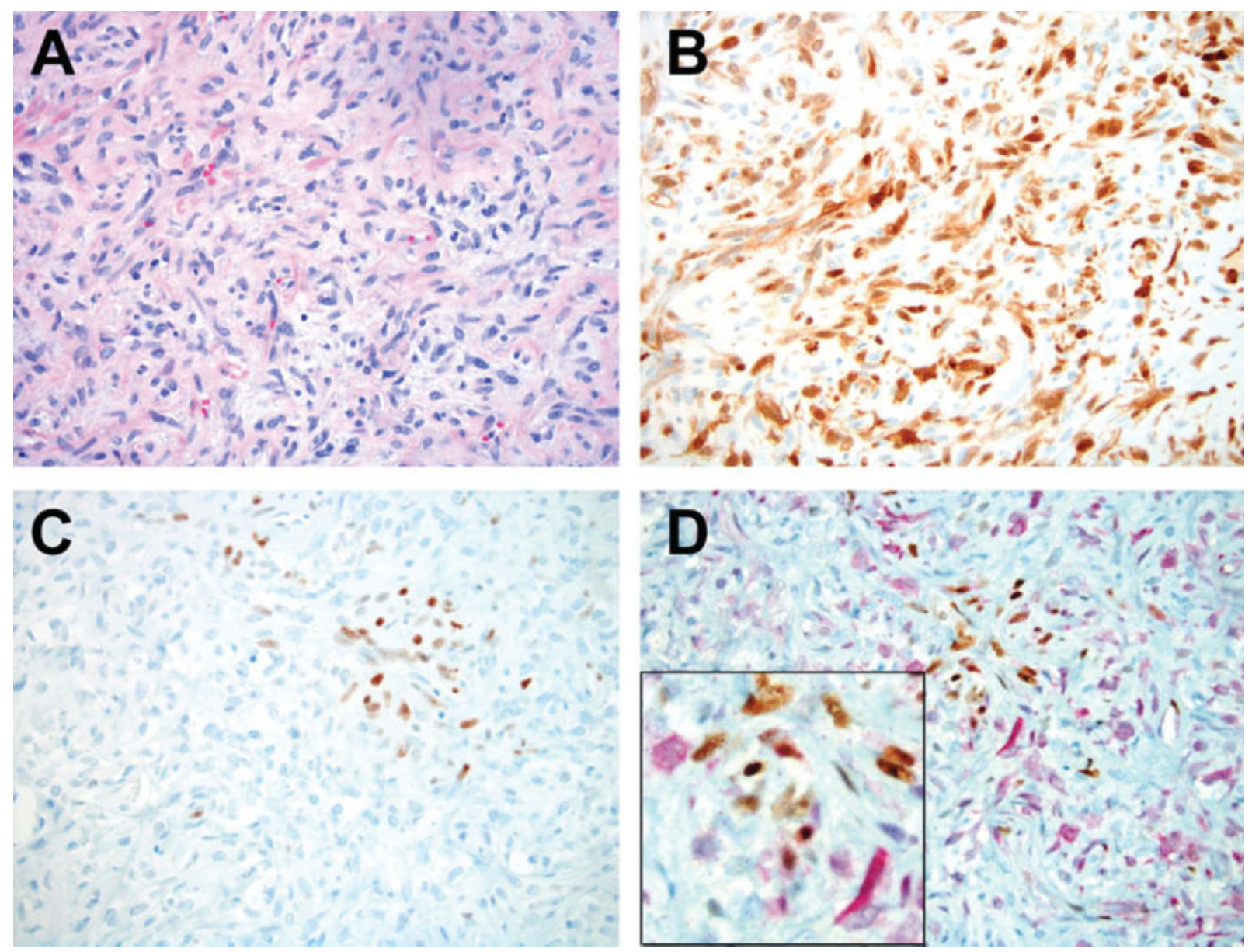

Fig. 3 p16 and EGR1 expressions in SFT/HPCs [(A): Hematoxylin and eosin (H\&E) stain; (B): p16 IHC stain; (C): EGR1 IHC stain; (D): p16 and EGR1 dual IHC staining; note red cytoplasmic staining indicating p 16 immunopositivity, EGR1 positive cells show nuclear brown immunolabeling. Case \#8, 200× magnification; insert, 400× magnification]. 
In our study, we showed that CNS SFT/HPCs tend to be malignant and more likely to express the neuroendocrine marker synaptophysin. Chromogranin was immunonegative in all tumors, while CD56 showed immunopositivity in almost half of them $(n=11)$. None of these neuroendocrine markers show statistically significant correlation with malignancy, disease-free survival, or p16 expression. SST2A showed patchy staining in 2 malignant CNS SFT/HPCs, while all other tumors were negative. This could be useful for diagnostic purposes of extra-axial lesions. We also noted that non CNS tumors were larger than CNS SFT/HPCs. It could be explained by space limiting location in the skull and the prevalence of malignant CNS tumors.

IHC for STAT6 is a reliable marker to confirm the SFT/HPC destination. ${ }^{12,13}$ Our study, included the SFT/HPC cases that were confirmed by STAT6, IHC, or next generation sequencing to avoid the diagnostic ambiguity. NAB2-STAT6 fusion is the driver mutation of SFT/HPC and the fusion constitutively activates EGR1. ${ }^{2}$ As a potential downstream protein of EGR $1,{ }^{6} \mathrm{p} 16$, has been reported to be associated with malignancy and worse prognosis in multiple mesenchymal tumors. ${ }^{7-9}$ We found that high p16 expression (defined as positivity $\geq 50 \%$ tumor cells) is significantly associated with malignancy $(p=0.015)$ and shorter disease-free survival time $(p=0.045)$ among all tumors (-Fig. 2 ). The diseasefree survival analyses indicate that $\mathrm{p} 16$ expression is another useful prognostic marker for recurrence prediction. In our study, Ki67 proliferative index had varied from 2 to $45 \%$ and did not correlate with malignancy and/or disease-free survival statistically. This could be due to analyzing limited tumor field on microarray study.

Interestingly, EGR1 protein was not detected in every p16 positive tumor cell by IHC. This finding implies either p16 expression in SFT/HPC is independent of NAB2-STAT6 fusion-activated EGR1 signaling pathway, or EGR1 protein is not detected by IHC due to activation by NAB2-STAT6 fusion. It has been reported that NAB2-STAT6 fusion constitutively activates EGR1, but EGR1 protein expression is not increased in SFT/HPC tumors. ${ }^{2,14}$ EGR1 is a transcription factor that binds to DNA and influences p16 promoter activity. ${ }^{6}$ However, the failure to detect this protein by IHC in FFPE blocks is possibly could be due to epitopes that obscured or crosslinked in DNA, and the protein is nearer to its native form has parts of the amino acid chain folded inside. For further study, chromatin immunoprecipitation (ChIP) assay may be used to detect protein-DNA interaction that requires chromatin to be extracted and fragmented to expose protein from DNAprotein complexes before using specific antibodies against a target protein. ${ }^{15}$

As a cell cycle checkpoint protein p16 is considered to be a tumor suppressor and is down-regulated in a large number of tumors. Intriguingly, overexpression of p16 has also been described in several tumors. ${ }^{16}$ In HPV related tumors p16 overexpression will result in the inability to halt cell proliferation because of downstream Rb inactivation. ${ }^{17,18}$ Many other spindle-cell mesenchymal tumors are also positive for p16: (1) 89.5\% of well-differentiated liposarcomas; (2) $94.4 \%$ of dedifferentiated liposarcomas; (3) $77.8 \%$ of synovial sar- comas; and (4) significant percentages of angiosarcomas, rhabdomyosarcomas, leiomyosarcomas, and malignant fibrous histiocytomas. ${ }^{8,19,20}$ Thus, p16 is likely not a useful diagnostic marker for SFT/HPCs.

As a potential downstream protein of EGR $1,{ }^{6}$ p16 has a reported association with malignancy, and worse prognosis in multiple mesenchymal tumors. ${ }^{7-9}$ The results of this study demonstrate that high $\mathrm{p} 16$ expression is significantly associated with malignancy (-Fig. 1) and shorter disease-free survival time (- Fig. 2 ) in the SFT/HPC tumors. The diseasefree survival analyses indicate that p16 expression is another useful prognostic marker for recurrence prediction. Because p16 is a critical regulator of cell proliferation and is inactivated during the course of tumorigenesis, restoration of p16 function could provide therapeutic benefit. ${ }^{21}$

The major limitation of the current study is the singleinstitution nature of the patient population. The limited study population creates potential for exaggerated effect from false-positive and false-negative results. Future research should focus on recruiting a larger, multi-institutional population of patients for improved statistical power in evaluation of the diagnostic and prognostic effect of p16 in patients with SFT.

\section{Conclusion}

SFT/HPCs located in the CNS are more likely to be malignant than tumors of other sites. High p16 expression is associated with malignancy and shorter disease-free survival time in SFT/HPCs. The alteration of p16 overexpression as possible therapeutic venue should be further explored.

\section{Authors' Contributions}

Study design: Y.L., R.S.H., J.K.W., C.B.H., A.S.T., and K.A. Study conduct and data analysis: Y.L., K.A., and R.S.H. Manuscript preparation: Y.L., K.A., and R.S.H.

\section{Acknowledgment}

We thank Mushahid Asadi and Annette Shepard-Barry for excellent assistance with tissue microarray and histological techniques.

\section{References}

1 Doyle LA, Vivero M, Fletcher CD, Mertens F, Hornick JL. Nuclear expression of STAT6 distinguishes solitary fibrous tumor from histologic mimics. Mod Pathol 2014;27(03):390-395

2 Robinson DR, Wu YM, Kalyana-Sundaram S, et al. Identification of recurrent NAB2-STAT6 gene fusions in solitary fibrous tumor by integrative sequencing. Nat Genet 2013;45(02):180-185

3 Chmielecki J, Crago AM, Rosenberg M, et al. Whole-exome sequencing identifies a recurrent NAB2-STAT6 fusion in solitary fibrous tumors. Nat Genet 2013;45(02):131-132

4 Zwang Y, Oren M, Yarden Y. Consistency test of the cell cycle: roles for p53 and EGR1. Cancer Res 2012;72(05):1051-1054

5 Virolle T, Adamson ED, Baron V, et al. The Egr-1 transcription factor directly activates PTEN during irradiation-induced signalling. Nat Cell Biol 2001;3(12):1124-1128

6 Chaturvedi V, Cesnjaj M, Bacon P, et al. Role of INK4a/Arf locusencoded senescent checkpoints activated in normal and psoriatic keratinocytes. Am J Pathol 2003;162(01):161-170 
7 Honoki K, Stojanovski E, McEvoy M, et al. Prognostic significance of p16 INK4a alteration for Ewing sarcoma: a meta-analysis. Cancer 2007;110(06):1351-1360

8 Kammerer-Jacquet SF, Thierry S, Cabillic F, et al. Differential diagnosis of atypical lipomatous tumor/well-differentiated liposarcoma and dedifferentiated liposarcoma: utility of p16 in combination with MDM2 and CDK4 immunohistochemistry. Hum Pathol 2017;59:34-40

9 Gannon BR, Manduch M, Childs TJ. Differential Immunoreactivity of p16 in leiomyosarcomas and leiomyoma variants. Int J Gynecol Pathol 2008;27(01):68-73

10 Fletcher CDM, Bridge JA, Hogendoorn P, Mertens F. IARC/WHO Classification of Tumours of Soft Tissue and Bone. 4th ed. WHO; 2013:80-82

11 Louis DN, Perry A, Reifenberger G, et al. The 2016 World Health Organization classification of tumors of the central nervous system: a summary. Acta Neuropathol 2016;131(06):803-820

12 Schweizer L, Koelsche C, Sahm F, et al. Meningeal hemangiopericytoma and solitary fibrous tumors carry the NAB2-STAT6 fusion and can be diagnosed by nuclear expression of STAT6 protein. Acta Neuropathol 2013;125(05):651-658

13 Berghoff AS, Kresl P, Bienkowski M, et al. Validation of nuclear STAT6 immunostaining as a diagnostic marker of meningeal solitary fibrous tumor (SFT)/hemangiopericytoma. Clin Neuropathol 2017;36(02):56-59
14 Vogels RJ, Vlenterie M, Versleijen-Jonkers YM, et al. Solitary fibrous tumor - clinicopathologic, immunohistochemical and molecular analysis of 28 cases. Diagn Pathol 2014;9:224

15 Cai YH, Huang H. Advances in the study of protein-DNA interaction. Amino Acids 2012;43(03):1141-1146

16 Romagosa C, Simonetti S, López-Vicente L, et al. p16(Ink4a) overexpression in cancer: a tumor suppressor gene associated with senescence and high-grade tumors. Oncogene 2011;30(18): 2087-2097

17 Martin A, Baran-Marzak F, El Mansouri S, et al. Expression of p16/ INK4a in posttransplantation lymphoproliferative disorders. Am J Pathol 2000;156(05):1573-1579

18 Reuschenbach M, Waterboer T, Wallin KL, et al. Characterization of humoral immune responses against p16, p53, HPV16 E6 and HPV16 E7 in patients with HPV-associated cancers. Int J Cancer 2008;123(11):2626-2631

19 Knösel T, Altendorf-Hofmann A, Lindner L, et al. Loss of p16(INK4a) is associated with reduced patient survival in soft tissue tumours, and indicates a senescence barrier. J Clin Pathol 2014;67(07):592-598

20 Shim BY, Yoo J, Lee YS, Hong YS, Kim HK, Kang JH. Prognostic role of Rb, p16, Cyclin D1 proteins in soft tissue sarcomas. Cancer Res Treat 2010;42(03):144-150

21 Zhao R, Choi BY, Lee MH, Bode AM, Dong Z. Implications of genetic and epigenetic alterations of CDKN2A (p16(INK4a)) in cancer. EBioMedicine 2016;8:30-39 